Scientific Paper

\title{
Quantitative and dosimetric analysis for treating synchronous bilateral breast cancer using two radiotherapy planning techniques
}

\author{
Özlem MermutTa, ${ }^{\mathrm{a}}$, Aysun Ozsoy ATA ${ }^{\mathrm{b}}$, Didem CAN TRABULUS ${ }^{\mathrm{c}}$ \\ ${ }^{a}$ Department of Radiation Oncology, University of Health Sciences Istanbul Training and Research Hospital, Turkey \\ ${ }^{b}$ Department of Radiation Oncology, MNT Health Services Medical Physicist, University of Health Sciences Istanbul Training \\ and Research Hospital, Turkey \\ ${ }^{c}$ Clinic of General Surgery, University of Health Sciences Istanbul Training and Research Hospital, Turkey \\ ${ }^{*}$ E-mail address: mermutozlem@gmail.com
}

\begin{abstract}
Objective: We compared mono-isocenter and dual-isocenter plans in synchronous bilateral breast cancer (SBBC), which is defined as tumours occurring simultaneously in both breasts, and evaluated the effects of these differences in plans on organs-at-risk (OARs).

Materials and methods: We evaluated 10 women with early stage, nod negative (Tis-2N0M0) SBBC. The treatment dose was determined to be $50 \mathrm{~Gy}$. We used mean dose and $\mathrm{V}_{\mathrm{XGy}}$ to evaluate the OARs. To evaluate the effectiveness of treatment plans, Homogeneity index (HI), conformity index (CI) and sigma index (SI) and monitor units (MU) of monoisocenter (MIT) and dual-isocenter (DIT) plans were compared. During bilateral breast planning, for the single-centre plan, the isocenter was placed at the center of both breasts at a depth of 3-4 cm. For the two-center plan, dual-isocenters were placed on the right and left breasts.

Results: No significant difference between the techniques in terms of the scope of the target volume was observed. Statistically significant results were not achieved in MIT and DIT plans for OARs. Upon comparing MIT and DIT, the right-side monitor unit $(\mathrm{MU})$ value in DIT $(\mathrm{p}=0.011)$ was statistically significantly lower than that in MIT. Upon comparing right-left side MIT and DIT, the MU value $(\mathrm{p}=0.028)$ was significantly lower in DIT than MIT.

Conclusion: SBBC irradiation is more complex than unilateral breast radiotherapy. No significant difference between both techniques and OARs was observed. However, we recommend MIT as a priority technique due to the ability to protect OARs, ease of administration during treatment, and the fact that the patient stays in the treatment unit for a shorter period of time.
\end{abstract}

Key words: dosimetric comparison; mono-isocenter; dual-isocenter; synchronous bilateral breast cancer.

\section{Introduction}

Breast cancer is the most commonly diagnosed malignancy in the population of women globally. ${ }^{1}$ With the increase in the incidence of breast cancer and life expectancy, the incidence of developing bilateral breast cancer has also increased. ${ }^{1}$ The incidence of synchronous bilateral breast cancer (SBBC) is between $0.3 \%-12 \%$ of breast cancers. ${ }^{2}$ The frequency of SBBC in our clinic is $2.2 \%$.

The surgical treatment method in SBBC is selected according to the location of the tumour in the breast, size, breast-tumour ratio, age of patient, requirements of the patient, and whether it has spread to the axillary lymph nodes. Breast-conserving surgery (BCS) was shown to provide results comparable to mastectomy. ${ }^{3}$ For patients with early-stage breast cancer, radiotherapy to the protected breast after breast protective surgery reduces the risk of local recurrence and cancer-related death. ${ }^{4}$ Synchronous irradiation of both breasts after BCS in patients with SBBC is a challenge and complicated due to the need for minimizing dose to the heart and lungs.

To the best of our knowledge, this study is the first to compare mono-isocenter and dual-isocenter 3-D conformal field-in-field (FinF) plans and to evaluate the effects of these differences in plans on organs-at-risk.

\section{Material and methods}

We evaluated 10 women who underwent adjuvant radiotherapy after breast-conserving surgery with a diagnosis in the early stage, nod negative (Tis-2NOM0) SBBC. The median age of the patients was 57 (36-69) years, and the follow-up time was 41 months. We did not evaluate the boost plans because the tumour locations and sizes were different. To remain stable during the treatment, each patient was given a special vacuum bag that could be fixed to the breast surface while the patients were tied together in the back position, with their hands on the head.

(C) 2021 Özlem Mermut, Aysun Ozsoy Ata, Didem Can Trabulus. This is an open access article licensed under the Creative Commons Attribution-NonCommercialNoDerivs License (http://creativecommons.org/licenses/by-nc-nd/4.0/). 
Computed tomography (Siemens Somatom Spirit) scans were acquired in free-breathing mode with $3 \mathrm{~mm}$ thick axial sections. The scans were carried out to cover the entire lung volume and liver, from the sixth cervical to the second lumbar vertebra. The treatment dose was determined at 50 Gy (2 Gy/25 fraction), calculated using $6 \mathrm{MV}$ and/or 6-18 MV mixed photons. We used the mean dose and $\mathrm{V}_{\mathrm{XGy}}$ to evaluate organs-at-risk. The average dose of $V_{5 G y}, V_{20 G y}, V_{40 G y}$, and $D_{\text {mean }}$ was compared for the lung; the average dose of $\mathrm{V}_{10 \mathrm{~Gy}}, \mathrm{~V}_{25 \mathrm{~Gy}}, \mathrm{~V}_{40 \mathrm{~Gy}}$, and $\mathrm{D}_{\text {mean }}$ was compared for the heart, and the average dose of $V_{5 G y}, V_{30 G y}$, and $D_{\text {mean }}$ was compared for the liver. To evaluate the effectiveness of treatment plans, Homogeneity index (HI), conformity index (CI) and sigma index (SI) and monitor units (MU) of mono-isocenter and dual-isocenter plans were compared. PTV was aimed to receive $95 \%$ of the prescribed dose.

We obtained approval for this study from the ethics committee (15.03.2019/1742) of our hospital. Written consent was obtained from the patients.

\section{Target volume and organs-at-risk}

All the targets and critical organs identified in the planning were contoured by the same radiation oncologist according to ESTRO guidelines. Although clinical target volume (CTV) covered the entire breast, planning target volume (PTV) was obtained by giving an $8 \mathrm{~mm}$ margin in each direction. PTVs were created by pulling $5 \mathrm{~mm}$ below the skin surface, except for the ribs.

The heart was contoured from the level of the pulmonary trunk to include the apex, ventricles, atria, cardiac septum, and pericardium, to the level of entry and exit of the major vessels (descendant-ascendant aorta and vena cava). The left and right lungs and the liver were contoured separately. There is no certain treatment protocol for SBBC. Data from BBC patient trials were used (for mean lung dose $<15 \mathrm{~Gy}$, for bilateral lungs $\mathrm{V}_{20 \mathrm{~Gy}}<$ $30 \%$, for heart $\mathrm{V}_{25 \mathrm{~Gy}}<20 \%$ ) rather than dose suggestions used in unilateral breast radiation for dose suggestions for OARs. ${ }^{5}$

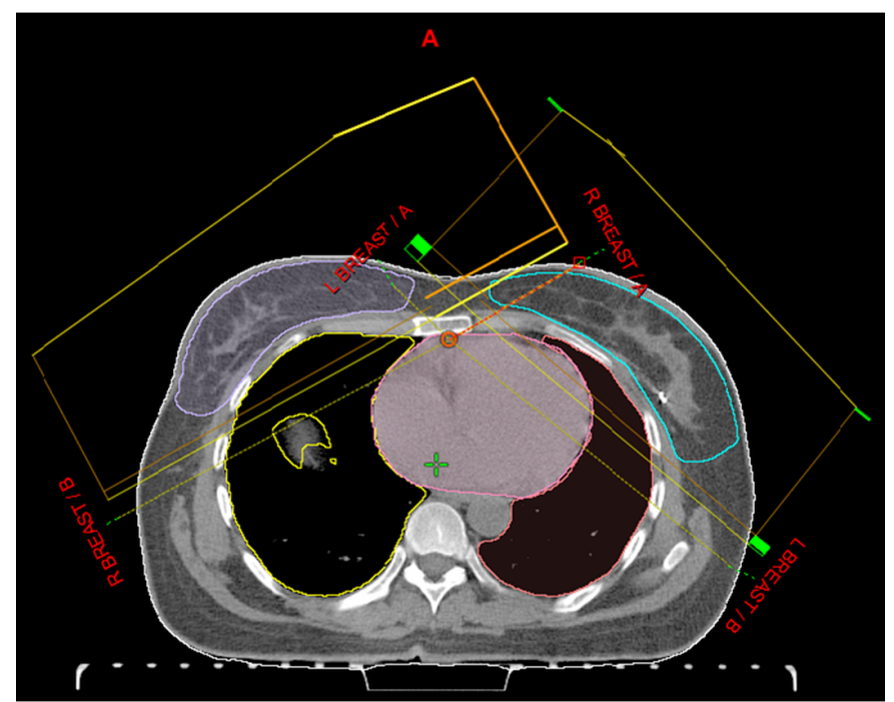

Figure 1a. Mono-isocenter fields view of plans for a patient.
The plans were created by the same medical physics expert using the Varian Eclipse treatment planning system (TPS) (version 10.0; Anisotropic Analytical Algorithm (AAA), Varian Medical Systems, Palo Alto, CA, USA).

\section{Planning Techniques}

During bilateral breast planning, for the single-centre plan, the isocenter was placed at the center of both breasts at a depth of $3-4 \mathrm{~cm}$ from the skin to the sternum. The center was determined $3-4 \mathrm{~cm}$ below the vertical axis by taking the midpoint of the patient's breasts on both the lateral and longitudinal axis (Figure 1a). For the two-center plan, dual-isocenters were placed on the right and left breasts. The mean depth of the breasts was chosen at two separate points on the right and left longitudinal axis, vertically in both breasts for dual isocenter (Figure 1b).

The only difference between the two centers ( $\mathrm{x}, \mathrm{y}$, and $\mathrm{z}$ ) was in the lateral ( $\mathrm{x}$ ) plane for easy treatment. When determining the inner tangential and outer tangential fields, care was taken that the fields did not overlap. After the center was determined in bilateral tangential breast planning, the organs-at-risk were protected by multi-leaf collimators (MLC) by taking advantage of beam eye view (BEV). Overlapping areas were not allowed in the plans. In order to avoid hot dose zones, attention was taken not to overlap the field entrances. To make homogeneous dose distribution of beams used against each other, the weights were adjusted. Hot dose zones above $110 \%$ of treatment dose in TPS were plotted using the 'convert isodose level to structure' feature. Designed hot spot zones were closed with MLCs and subfields were calculated. Dose homogeneity in volume was evaluated with cDVH.

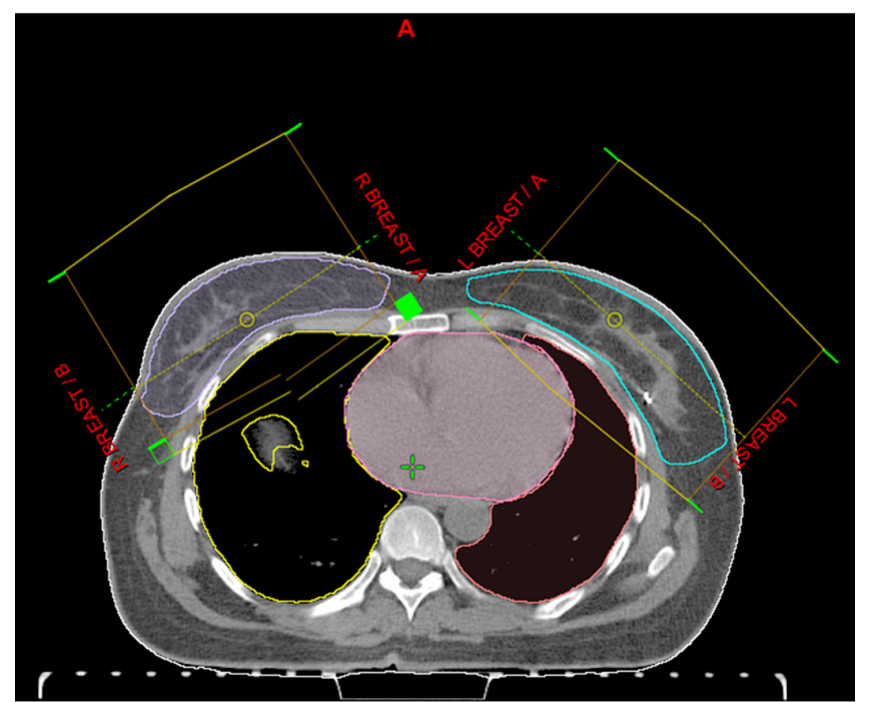

Figure 1b. Dual-isocenter fields view of plans for a patient. 


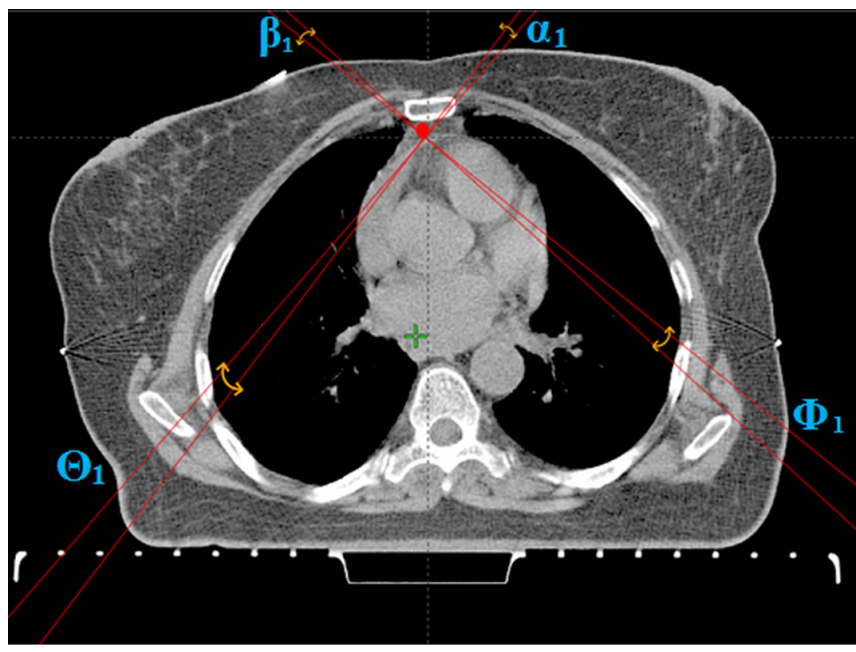

Figure 2a. Ranges of inner and outer tangential angles used in MIT during planning. (Angles $\Theta_{1}=222^{\circ}-242^{\circ}, \beta_{1}=309^{\circ}-319^{\circ}, \alpha_{1}=38^{\circ}$ $\left.60^{\circ}, \Phi_{1}=124^{\circ}-135^{\circ}\right)$

The degree of angles used in inner tangential areas for MIT during planning was $38^{\circ}-60^{\circ}$ degrees on the right; $309^{\circ}-319^{\circ}$ degrees on the left and $222^{\circ}-242^{\circ}$ degrees on the right in outer tangential areas; $124^{\circ}-135^{\circ}$ degrees on the left (Figure 2a).

The degree of angles used in inner tangential areas for DIT during planning was $45^{\circ}-57^{\circ}$ degrees on the right; $306^{\circ}-317^{\circ}$ degrees on the left and $220^{\circ}-239^{\circ}$ degrees on the right in outer tangential areas; $129^{\circ}-140^{\circ}$ degrees on the left (Figure 2b).

\section{Plan Analysis}

The plans were analysed using cDVH (cumulative dose-volume histogram) and dDVH (differential dose-volume histogram). Homogeneity index (HI), conformity index (CI) and sigma index (SI) were defined to describe the quality of plans as follows:

Homogeneity index (HI): HI was evaluated according to ICRU $83 .{ }^{6} \mathrm{D} 2 \%$, D50\%, and D98\% mean the doses of $2 \%, 50 \%$, and $98 \%$ volume of the PTV.

$H I=\frac{D_{2 \%}-D_{98 \%}}{D_{50 \%}}$

Eq. 1

Conformity Index (CI): TV is the target volume. $\mathrm{V}_{\mathrm{PIV}}$ is the treatment volume of the body receiving $95 \%$ of the prescribed dose; $\mathrm{V}_{\mathrm{TV}}$ is the volume of PTV; and $\mathrm{TV}_{\mathrm{PV}}$ is the target volume covered by the prescription isodose. CI values are between one and zero; and bigger CI value identifies a stronger conformity degree. ${ }^{7}$ The cDVH of the plans was used when calculating HI and CI. CI values close to 1 identify extremely uniform coverage.

$C I=\frac{T V_{p v} \times T V_{p v}}{V_{P I V} \times V_{T V}}$

Eq. 2

Sigma Index (SI): Homogeneity of dose within the target volume was assessed using the Sigma index, as defined by the standard deviation of the normalised differential curve of PTV

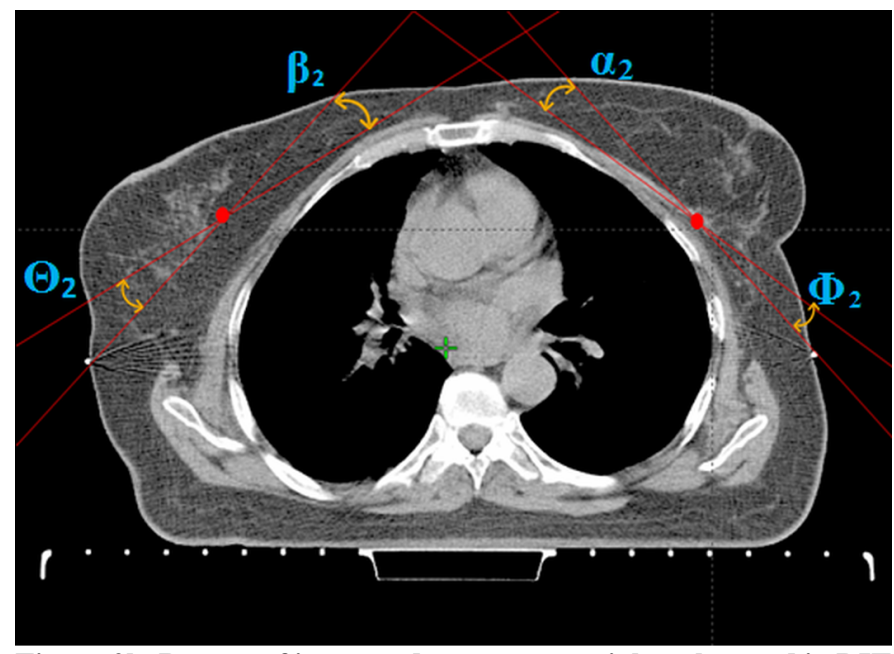

Figure 2b. Ranges of inner and outer tangential angles used in DIT during planning. (Angles $\Theta_{2}=220^{\circ}-239^{\circ}, \beta_{2}=45^{\circ}-57^{\circ}, \alpha_{2}=306^{\circ}$ $317^{\circ}, \Phi_{2}=129^{\circ}-140^{\circ}$ )

volume, where $D_{S D}$ represents the standard deviation of the dose, $v_{i}$ is the $i$ th volume element receiving a dose of at least $\left(D_{i}\right)$, and $V$ is the total volume of PTV. $D_{\text {mean }}$ is the mean dose of PTV. If the SI value is near zero, then the PTV has superior dose homogeneity within the target. ${ }^{8}$ The dDVH of plans was used when calculating SI.

$$
S \dot{I}=D_{S D}=\sqrt{\left(\sum\left(D_{i}-D_{\text {mean }}\right)^{2} \times v_{i} / V\right)}
$$

\section{Statistical method}

Mean, standard deviation, median lowest, highest, frequency, and ratio values were used in the descriptive statistics of the data. The distribution of variables was measured using the Kolmogorov-Smirnov test. The Wilcoxon test was used in the analysis of dependent quantitative data. SPSS 26.0 was used for analyses.

\section{Results}

Four main fields (left/right, tangential inner/outer ) and 2 and/or 3 subfields were used for each plan. The median lung volumes were $1141 \mathrm{~cm}^{3}$ left side and $1275 \mathrm{~cm}^{3}$ right side; the median heart volume was $648 \mathrm{~cm}^{3}$ and the median liver volume was $1559 \mathrm{~cm}^{3}$.

Compared with the mono-isocenter technique (MIT), $\mathrm{D}_{\text {mean }}(p$ $=0.594), \mathrm{CI}(p=0.052)$, and SI $(p=0.670)$ were higher but not statistically significantly different to those of the dual-isocenter technique (DIT) on the right side. In the DIT, the ratio of CI was closer to 1 ( $p=0.052)$. The value of HI $(p=0.905)$ was the same for both techniques. The MU value on the right side for DIT ( $p$ $=0.011$ ) was statistically significantly lower than that for MIT. Upon comparing MIT with DIT on the left side, $\mathrm{D}_{\text {mean }}(p=$ $0.475)$, and CI ( $p=0.575)$ were higher but not statistically significantly different. The HI value $(p=0.906)$ was the same for both techniques. The SI $(p=0.438)$ and MU values ( $p=$ 0.154 ) were lower and not statistically significantly different. 
Upon comparing MIT and DIT on the right-left side, $\mathrm{D}_{\text {mean }}(p=$ $0.508)$, and CI $(p=0.052)$ were found to be high but not statistically significantly different. The HI $(p=0.307)$ and SI $(p$ $=0.887$ ) values were the same for both techniques and not statistically significantly different. The MU value $(p=0.028)$ was significantly lower for the dual-isocenter than the monoisocenter plan (Table 1).

Comparing MIT and DIT for the left lung, values for $\mathrm{D}_{\text {mean }}(p$ $=0.959), \mathrm{V}_{5 \mathrm{~Gy}}(p=0.674)$, and $\mathrm{V}_{40 \mathrm{~Gy}}(p=0.919)$ were lower, but not statistically significantly different in MIT. $\mathrm{V}_{20 \mathrm{~Gy}}$ value ( $p$ $=0.508$ ) was the same for both techniques, but not statistically significantly different.

Comparing MIT and DIT for the right lung, values for $\mathrm{D}_{\text {mean }}$ $(p=0.241), \mathrm{V}_{20 \mathrm{~Gy}}(p=0.358)$, and $\mathrm{V}_{40 \mathrm{~Gy}}(p=0.221)$ were lower, but not statistically significantly different in MIT. $\mathrm{V}_{5 \mathrm{~Gy}}$ value $(p$ $=0.168$ ) was higher, but not statistically significantly different.

Comparing MIT and DIT for both lungs, the values for $\mathrm{D}_{\text {mean }}$ $(p=0.386), \mathrm{V}_{5 \mathrm{~Gy}}(p=0.153)$, and $\mathrm{V}_{40 \mathrm{~Gy}}(p=0.083)$ were lower, but not statistically significantly different in MIT. $\mathrm{V}_{20 \mathrm{~Gy}}$ value $(p$ $=0.721$ ) was the same for both techniques but not statistically significantly different.

Comparing MIT and DIT for the heart, the values for $\mathrm{D}_{\text {mean }}(p$ $=0.646), \mathrm{V}_{10 \mathrm{~Gy}}(p=0.385)$, and $\mathrm{V}_{40 \mathrm{~Gy}}(p=0.308)$ were higher, but not statistically significantly different in MIT. $\mathrm{V}_{25 \text { Gy }}$ value ( $p$ $=1.000$ ) was lower, but not statistically significantly different in MIT.

Comparing MIT and DIT for the liver, the values for $\mathrm{D}_{\text {mean }}(p$ $=0.285)$ and $\mathrm{V}_{5 \mathrm{~Gy}}(p=0.878)$ were lower, but not statistically significantly different in MIT. $\mathrm{V}_{30 \mathrm{~Gy}}$ value $(p=0.441)$ was higher, but not statistically significantly different in MIT (Table 2).

\section{Discussion}

In SBBC treatment planning, bilateral PTV creates a large irradiation area. The lungs and heart are at greater risk in bilateral PTV than in unilateral breast cancer. Important differences in the form and width of the breast have been observed among patients. If the patient is obese or the volume of the lungs is small, the lungs and heart are exposed to higher doses.

The prevention of radiation-induced lung toxicity is particularly important in SBBC. The notified incidence of radiation pneumonia in the literature ranges from $0 \%-80 \%$, based on the radiotherapy technique used., ${ }^{\mathbf{9} 10}$ The $\mathrm{V}_{20 \mathrm{~Gy}}$ dose of the lungs is associated with high radiation pneumonia, and dose restrictions are used for the evaluation of treatment plans. ${ }^{11,12}$ In our study, we found the mean lung doses of 9.9 Gy for MIT and 10.4 Gy for DIT. The median $\mathrm{V}_{20 \mathrm{~Gy}}$ value was $18.2 \%$ for bilateral lungs. For both techniques, the dose for the lungs was acceptable.
Table 1. Planning target volume, conformity index, homogeneity index, sigma index, and monitor unit variables for the right, left, and bilateral sides.

\begin{tabular}{|c|c|c|c|c|c|}
\hline & \multicolumn{2}{|c|}{ Mono-isocenter (MIT) } & \multicolumn{2}{|c|}{ Dual isocenter (DIT) } & \multirow{2}{*}{$\begin{array}{c}p \\
\text { value }\end{array}$} \\
\hline & Mean \pm sd & Median & Mean \pm sd & Median & \\
\hline \multicolumn{6}{|l|}{ Right side } \\
\hline $\mathrm{TV}\left(\mathrm{PTV} \mathrm{cm}^{3}\right)$ & $912.1 \pm 417.9$ & 987 & $912.1 \pm 417.9$ & 987 & 1.000 \\
\hline D mean (Gy) & $52.4 \pm 0.22$ & 52.4 & $52.3 \pm 0.18$ & 52.3 & 0.594 \\
\hline CI & $0.54 \pm 0.08$ & 0.55 & $0.51 \pm 0.08$ & 0.54 & 0.052 \\
\hline HI & $0.12 \pm 0.01$ & 0.12 & $0.12 \pm 0.01$ & 0.12 & 0.905 \\
\hline SI & $3.13 \pm 0.31$ & 3.25 & $3.10 \pm 0.28$ & 3.10 & 0.670 \\
\hline MU & $239.5 \pm 6.8$ & 238.5 & $236.4 \pm 7.2$ & 235 & 0.011 \\
\hline \multicolumn{6}{|l|}{ Left side } \\
\hline $\mathrm{TV}\left(\mathrm{PTV} \mathrm{cm}^{3}\right)$ & $915.9 \pm 363.8$ & 846 & $915.9 \pm 363.8$ & 846 & 1.000 \\
\hline D mean (Gy) & $52.4 \pm 0.18$ & 52.4 & $52.3 \pm 0.29$ & 52.3 & 0.475 \\
\hline CI & $0.56 \pm 0.05$ & 0.55 & $0.55 \pm 0.05$ & 0.54 & 0.575 \\
\hline HI & $0.12 \pm 0.01$ & 0.12 & $0.12 \pm 0.01$ & 0.12 & 0.906 \\
\hline SI & $3.05 \pm 0.36$ & 2.95 & $3.14 \pm 0.36$ & 3.20 & 0.438 \\
\hline MU & $241 \pm 8.5$ & 237.5 & $238.3 \pm 6.9$ & 238 & 0.154 \\
\hline \multicolumn{6}{|c|}{ Right+Left side } \\
\hline $\mathrm{TV}\left(\mathrm{PTV} \mathrm{cm}^{3}\right)$ & $1728 \pm 897.5$ & 1879 & $1728 \pm 897.5$ & 1879 & 1.000 \\
\hline D mean (Gy) & $52.4 \pm 0.17$ & 52.4 & $52.3 \pm 0.21$ & 52.3 & 0.508 \\
\hline CI & $0.55 \pm 0.06$ & 0.54 & $0.53 \pm 0.06$ & 0.53 & 0.052 \\
\hline HI & $0.12 \pm 0.01$ & 0.12 & $0.12 \pm 0.01$ & 0.12 & 0.307 \\
\hline SI & $3.13 \pm 0.29$ & 3.15 & $3.12 \pm 0.21$ & 3.15 & 0.887 \\
\hline MU & $460.4 \pm 68.4$ & 477.5 & $454.7 \pm 70.5$ & 471 & 0.028 \\
\hline
\end{tabular}

Table 2. OAR variables.

\begin{tabular}{|c|c|c|c|c|c|}
\hline & \multicolumn{2}{|c|}{ Mono-isocenter (MIT) } & \multicolumn{2}{|c|}{ Dual isocenter (DIT) } & \multirow{2}{*}{$\begin{array}{c}p \\
\text { value }\end{array}$} \\
\hline & Mean \pm sd & Median & Mean \pm sd & Median & \\
\hline \multicolumn{6}{|l|}{ Left lung } \\
\hline Volume $\left(\mathrm{cm}^{3}\right)$ & $1193.3 \pm 335$ & 1141 & $1193.3 \pm 335$ & 1141 & 1.000 \\
\hline Dmean (Gy) & $9.7 \pm 0.23$ & 8.8 & $9.6 \pm 0.23$ & 9 & 0.959 \\
\hline $\mathrm{V}_{5 \mathrm{~Gy}}(\%)$ & $31.1 \pm 6.1$ & 30.5 & $31.7 \pm 6.9$ & 32 & 0.674 \\
\hline $\mathrm{V}_{20 \mathrm{~Gy}}(\%)$ & $17 \pm 4.9$ & 15.7 & $16.6 \pm 5$ & 15.7 & 0.508 \\
\hline $\mathrm{V}_{40 \mathrm{~Gy}}(\%)$ & $11.13 \pm 4.6$ & 9.5 & $11.5 \pm 4.3$ & 10.9 & 0.919 \\
\hline \multicolumn{6}{|l|}{ Right lung } \\
\hline Volume $\left(\mathrm{cm}^{3}\right)$ & $1411.2 \pm 377.7$ & 1275 & $1411.2 \pm 377.7$ & 1275 & 1.000 \\
\hline Dmean (Gy) & $11.1 \pm 0.28$ & 11.1 & $11.6 \pm 0.24$ & 11.5 & 0.241 \\
\hline $\mathrm{V}_{5 \mathrm{~Gy}}(\%)$ & $35.8 \pm 8.9$ & 35.5 & $37.2 \pm 7.8$ & 35 & 0.168 \\
\hline $\mathrm{V}_{20 \mathrm{~Gy}}(\%)$ & $20.2 \pm 5.8$ & 20.1 & $20.8 \pm 4.9$ & 21.1 & 0.358 \\
\hline $\mathrm{V}_{40 \mathrm{~Gy}}(\%)$ & $14.1 \pm 5$ & 13.9 & $15 \pm 4.3$ & 15.1 & 0.221 \\
\hline \multicolumn{6}{|l|}{ Heart } \\
\hline Volume $\left(\mathrm{cm}^{3}\right)$ & $630 \pm 145$ & 648 & $630 \pm 145$ & 648 & 1.000 \\
\hline Dmean (Gy) & $5.06 \pm 0.83$ & 5.2 & $5.03 \pm 0.60$ & 4.9 & 0.646 \\
\hline $\mathrm{V}_{10 \mathrm{~Gy}}(\%)$ & $5.9 \pm 1.9$ & 6.1 & $5.5 \pm 1.3$ & 5.5 & 0.385 \\
\hline $\mathrm{V}_{25 \mathrm{~Gy}}(\%)$ & $3.8 \pm 1.2$ & 3.9 & $3.9 \pm 0.9$ & 4 & 1.000 \\
\hline $\mathrm{V}_{40 \mathrm{~Gy}}(\%)$ & $8.3 \pm 2.3$ & 8.8 & $7.6 \pm 1.7$ & 7.6 & 0.308 \\
\hline \multicolumn{6}{|l|}{ Liver } \\
\hline Volume $\left(\mathrm{cm}^{3}\right)$ & $1486.4 \pm 367.5$ & 1559 & $1486.4 \pm 367.5$ & 1559 & 1.000 \\
\hline Dmean (Gy) & $4.3 \pm 0.20$ & 3.5 & $4.4 \pm 0.15$ & 3.7 & 0.285 \\
\hline $\mathrm{V}_{5 \mathrm{~Gy}}(\%)$ & $11.5 \pm 5.7$ & 9 & $11.3 \pm 4.4$ & 9.7 & 0.878 \\
\hline $\mathrm{V}_{30 \mathrm{~Gy}}(\%)$ & $5.8 \pm 3.8$ & 4.1 & $5.4 \pm 2.7$ & 4 & 0.441 \\
\hline \multicolumn{6}{|l|}{ Total lungs } \\
\hline Volume $\left(\mathrm{cm}^{3}\right)$ & $2604.9 \pm 676$ & 2441 & $2604.9 \pm 676$ & 2441 & 1.000 \\
\hline Dmean (Gy) & $10.5 \pm 0.21$ & 9.9 & $10.8 \pm 0.18$ & 10.4 & 0.386 \\
\hline $\mathrm{V}_{5 \mathrm{~Gy}}(\%)$ & $33.8 \pm 6.4$ & 32.5 & $34.9 \pm 6.2$ & 35 & 0.153 \\
\hline $\mathrm{V}_{20 \mathrm{~Gy}}(\%)$ & $18.9 \pm 4.3$ & 18.2 & $19.1 \pm 3.8$ & 18.2 & 0.721 \\
\hline $\mathrm{V}_{40 \mathrm{~Gy}}(\%)$ & $12.7 \pm 3.7$ & 12.3 & $13.5 \pm 3.2$ & 12.8 & 0.083 \\
\hline
\end{tabular}


The heart is a critical organ involved in breast radiotherapy. Cardiovascular disease after radiotherapy has become the main cause of non-breast cancer deaths in patients with breast cancer. ${ }^{13}$ For this reason, cardiotoxicity induced by radiotherapy is an important problem. Pignol et al. reported that that the lowest cardiac doses were delivered in the radiotherapies performed through IMRT and 3D-CRT planning. ${ }^{14}$ In Darby's study, for every increase of $1 \mathrm{~Gy}$ in the mean radiation dose to the heart, the rate of ischaemic heart disease increased by $7.4 \% .^{15}$ Therefore, decreasing the mean heart dose is vital for decreasing long-term cardiotoxicity. Sun et al. reported that in IMRT, VMAT, HT, and IMPT (proton) dosimetry for SBBC, IMRT-MIT plans provided higher dose homogeneity and lower heart dose than DIT. In the same study, VMAT-DIT plans demonstrated higher compatibility and significantly decreased lung, heart, LV, and LAD doses than VMAT-MIT plans. ${ }^{16}$ In our study, the $\mathrm{V}_{25 \mathrm{~Gy}}$ value was $3.9 \%$ in MIT and $4 \%$ in DIT and the dose for the heart was acceptable.

Patients with radiation-induced liver disease (RILD) take a significantly higher mean dose to the liver; RILD incidence rate was found to be $<5 \%$ when the entire liver dose was $<30-32$ Gy. ${ }^{17}$ Kim et al. used multiple techniques and reported that IMPT plans significantly reduced the mean dose to the liver, and HT plans increased the mean dose compared to other plans ( $\mathrm{p}<$ 0.01). HT plans showed the upper liver dose, but $\mathrm{D}_{\text {mean }}$ was $<5$ Gy. ${ }^{18}$ The $D_{\text {mean }}$ value for the liver was found to be $3.5 \mathrm{~Gy}$ in MIT and 3.7 Gy in DIT. The $\mathrm{V}_{30 \mathrm{~Gy}}$ value for the liver was $<4 \%$ in MIT and $<4.1 \%$ in DIT, and almost identical in both techniques. Thus, for liver protection, we recommend using both techniques.

Kim et al. reported that $\mathrm{CI}$ and $\mathrm{HI}$ values are superior with IMRT plans in MIT for PTV's. ${ }^{18}$ In our study, CI, HI, and SI values did not significantly differ for MIT and DIT.

In Boman et al. SBBC study with VMAT, MU times were found more in DIT plans than in MIT plans, unlike our study. ${ }^{19}$ In our study for MIT, center remains under the jaws. The center of the beam area and the center of the treatment area are not the same places, so normalization is higher, which increases MU. In DIT, the center is the same as the center of treatment and does not remain below the collimators. In the VMAT treatment, the field center does not close for both techniques.
Yavas's, Ohashi's, and Onal's studies have revealed dosimetric superiority of the FinF radiotherapy technique in breast cancer radiotherapy. ${ }^{\mathbf{2 0 - 2 2}}$ The FinF technique provides a more homogeneous dose distribution at the target volume than the 3DCRT technique. .1,22 $^{2}$

Increasing the number of subfields is necessary to reduce target-volume hot spots. The use of subfields is essential to eliminate hot spot areas in isodose curves and to provide better dose distribution in target volumes, as shown in Tanaka's study. $^{23}$

\section{Conclusion}

Compared to unilateral breast radiotherapy, SBBC irradiation is more complex, simultaneously affects both the lungs and the heart, and has a wider distribution of treatment volumes. The treatment areas in MIT and DIT are similar and no significant difference between the two techniques is expected.

An important problem in treatment using different isocenters is the undesirable increase in the dose at the affected site. In our study, we found a statistically significant difference between MIT and DIT on both sides and the right side in MU doses. Probably because the central axis is more close due to the given gantry slope and normalisation is increased for the winding of the dose.

DIT treatments necessitate additional work and time during the radiotherapy session. Because of the movement of the treatment bed, the therapist needs to enter the treatment room and conduct the lateral shift manually. MIT is preferred if the patient is to be treated in a shorter duration without being moved and without being laterally shifted to the table. Moreover, the MIT set up is easy.

If the patient is asked to receive less MU in total, DIT may be preferred. Hot or cold spots may occur when DIT is also shifting laterally to the patient. As shown in this study, there is no difference in both techniques and OAR's and acceptable plans can be achieved for SBBC treatment.

\section{References}

1. Bray F, Ferlay J, Soerjomataram I, Siegel RL, Torre LA, Jemal A. Global cancer statistics 2018: GLOBOCAN estimates of incidence and mortality worldwide for 36 cancers in 185 countries. CA Cancer J Clin. 2018;68(6):394-424. https://doi.org/10.3322/caac.21492

2. Krishnappa R, Chikaraddi SB, Deshmane V. Primary synchronous bilateral breast cancer. Indian J Cancer. 2014;51(3):256-258. https://doi.org/10.4103/0019-509X.146762

3. Irvine T, Allen DS, Gillett C, Hamed H, Fentiman IS. Prognosis of synchronous bilateral breast cancer. Br J Surg. 2009;96(4):376380. https://doi.org/10.1002/bjs.6553

4. Early Breast Cancer Trialists' Collaborative Group (EBCTCG), Darby S, McGale P, et al. Effect of radiotherapy after breast-conserving surgery on 10-year recurrence and 15-year breast cancer death: meta-analysis of individual patient data for 10,801 women in 17 randomised trials. Lancet. 2011;378(9804):1707-1716. https://doi.org/10.1016/S0140-6736(11)61629-2 
5. Nicolini G, Clivio A, Fogliata A, Vanetti E, Cozzi L. Simultaneous integrated boost radiotherapy for bilateral breast: A treatment planning and dosimetric comparison for volumetric modulated arc and fixed field intensity modulated therapy. Radiat Oncol. 2009;4:27. https://doi.org/10.1186/1748-717X-4-27

6. Hodapp N. ICRU Report 83 Prescribing, Recording, and Reporting Photon-Beam Intensity-Modulated Radiation Therapy (IMRT). Strahlenther Onkol. 2012;188:97-99. https://doi.org/10.1007/s00066-011-0015-x

7. Feuvret L, Noel G, Mazeron JJ, Bey P. Conformity index: a review. Int J Radiat Oncol Biol Phys. 2006;64:333-342. https://doi.org/10.1016/j.ijrobp.2005.09.028

8. Yoon M, Park SY, Shin D, et al. A new homogeneity index based on statistical analysis of the dose-volume histogram. J Appl Clin Med Phys. 2007;8(2):9-17. https://doi.org/10.1120/jacmp.v8i2.2390

9. Ramella S, Trodella L, Mineo TC et al. Adding ipsilateral V20 and V30 to conventional dosimetric constraints predicts radiation pneumonitis in stage IIIA-B NSCLC treated with combined-modality therapy. Int J Radiat Oncol Biol Phys. 2010;76(1):110-115. https://doi.org/10.1016/j.ijrobp.2009.01.036

10. Song CH, Pyo H, Moon SH, Kim TH, Kim DW, Cho KH. Treatment-related pneumonitis and acute esophagitis in non-small-cell lung cancer patients treated with chemotherapy and helical tomotherapy. Int J Radiat Oncol Biol Phys. 2010;78(3):651-658. https://doi.org/10.1016/j.ijrobp.2009.08.068

11. Lind PA, Wennberg B, Gagliardi G, et al. ROC curves and evaluation of radiation-induced pulmonary toxicity in breast cancer. Int $\mathbf{J}$ Radiat Oncol Biol Phys. 2006;64:765-770. https://doi.org/10.1016/j.ijrobp.2005.08.011

12. Yorke ED, Jackson A, Rosenzweig KE, Braban L, Leibel SA, Ling CC. Correlation of dosimetric factors and radiation pneumonitis for non-small-cell lung cancer patients in a recently completed dose escalation study. Int J Radiat Oncol Biol Phys. 2005;63(3):672682. https://doi.org/10.1016/j.ijrobp.2005.03.026

13. Fan LL, Luo YK, Xu JH, He L, Wang J, Du XB. A dosimetry study precisely outlining the heart substructure of left breast cancer patients using intensity-modulated radiation therapy. J Appl Clin Med Phys. 2014;15(5):4624. https://doi.org/10.1120/jacmp.v15i5.4624

14. Pignol JP, Keller BM, Ravi A. Doses to internal organs for various breast radiation techniques--implications on the risk of secondary cancers and cardiomyopathy. Radiat Oncol. 2011;6:5. https://doi.org/10.1186/1748-717X-6-5

15. Darby SC, Ewertz M, McGale P, et al. Risk of ischemic heart disease in women after radiotherapy for breast cancer. N Engl J Med. 2013;368(11):987-998. https://doi.org/10.1056/NEJMoa1209825

16. Sun T, Lin X, Tong $\mathrm{Y}$ et al. Heart and cardiac substructure dose sparing in synchronous bilateral breast radiotherapy: A dosimetric study of proton and photon radiation therapy. Front Oncol. 2020;9:1456. https://doi.org/10.3389/fonc.2019.01456

17. Cheng JC, Wu JK, Huang CM et al. Radiation-induced liver disease after three-dimensional conformal radiotherapy for patients with hepatocellular carcinoma: dosimetric analysis and implication. Int J Radiat Oncol Biol Phys. 2002;54(1):156-162. https://doi.org/10.1016/s0360-3016(02)02915-2

18. Kim SJ, Lee MJ, Youn SM. Radiation therapy of synchronous bilateral breast carcinoma (SBBC) using multiple techniques. Med Dosim. 2018;43(1):55-68. https://doi.org/10.1016/j.meddos.2017.08.003

19. Boman E, Rossi M, Kapanen M. The robustness of dual isocenter VMAT radiation therapy for bilateral lymph node positive breast cancer. Phys Med. 2017 Dec;44:11-17. https://doi.org/10.1016/j.ejmp.2017.11.006

20. Yavas G, Yavas C, Acar H. Dosimetric comparison of whole breast radiotherapy using field in field and conformal radiotherapy techniques in early stage breast cancer. Int J Radiat Res. 2012;10:131-138.

21. Ohashi T, Takeda A, Shigematsu N, et al. Dose distribution analysis of axillary lymph nodes for three-dimensional conformal radiotherapy with a field-in-field technique for breast cancer. Int J Radiat Oncol Biol Phys. 2009;73(1):80-87. https://doi.org/10.1016/j.ijrobp.2008.04.003.

22. Onal C, Sonmez A, Arslan G, et al. Dosimetric comparison of the field-in-field technique and tangential wedged beams for breast irradiation. Jap J Radiol. 2012;30(3):218-226. https://doi.org/10.1007/s11604-011-0034-7

23. Tanaka H, Hayashi S, Hoshi H. Determination of the optimal method for the field-in-field technique in breast tangential radiotherapy. J Radiat Res. 2014;55(4):769-773. https://doi.org/10.1093/jrr/rrt233 